\title{
Análise da ocorrência de eventos adversos pós-vacinação em Gurupi-TO
}

\author{
Analysis of the occurrence of post-vaccination adverse events in Gurupi-TO
}

Alessandra Luciane Lauermann Rico'1, Karen Lohanny Mendes Soares², Denise Soares de Alcântara ${ }^{3}$

\section{RESUMO}

Introdução: $O$ evento adverso pós-vacinação pode ser compreendido como sendo algum sinal ou sintoma inesperado após a administração de algum tipo de imunobiológico, tais como febre, rubor, calor, enduração,anafilaxia, episódio hipotônico hiporresponsivo entre outros. Com isso de acordo com as reações apresentadas os casos de eventos adversos após vacinação devem ser notificados para serem devidamente investigados. Objetivo: Analisar a ocorrência de eventos adversos pósvacinação no município de Gurupi-TO. Metodologia:Trata-se de um estudo descritivo, documental de abordagem quantitativarealizado na Secretaria Municipal de Saúde(SEMUS) de Gurupi Tocantins. A população alvo deste estudo foram as fichas de Eventos Adversos Pós Vacinação do Sistema de Informação de Eventos Adversos Pós Vacinação (SI-EAPV) dos casos ocorridos no município de Gurupi no período de 2011 a 2017 por serem estes disponíveis nos arquivos da SEMUS. A amostra consistiu de 73 fichas de notificação de eventos adversos pós-vacinais (EAPV). Os dados foram analisados por meio de estatística descritiva. Resultados: Observou-se que a maioria das notificações era de crianças menores de um ano $36,99 \%$, e o sexo feminino predominou entre as notificações com $57,53 \%$. A combinação das vacinas VIP, Penta, VORH e Pnc10v foi a que mais causou eventos adversos $16,44 \%$, seguida pela vacina DT aplicada de forma isolada representando $6,85 \%$ dos casos. Os eventos adversos pós-vacinação mais notificados durante o período do estudo foram os sistêmicos febre $32,88 \%$ e náuseas/vômitos $20,55 \%$, a maioria foi considerada não grave e o esquema vacinal foi mantido em $34,25 \%$ dos casos. Conclusão: Concluiuse que apesar dos imunobiológicos apresentarem benefícios, também oferecem riscos de EAPV. No entanto a efetividade da prevenção que a vacina proporciona supera os riscos de se ter um evento adverso provocado por ela. Este estudo servirá como ponto de partida para novos estudos no estado do Tocantins agregando conhecimentos sobre $o$ assunto.

Descritores: Vacinação. Imuno. Notificação. Reação adversa.

\section{ABSTRACT}

Introduction: The post-vaccination adverse event may be understood to be some unexpected sign or symptom following the administration of some type of immunobiological, such as fever, flushing, heat, induration, anaphylaxis, hyporesponsive hypotonic episode among others. Thus according to the reactions presented the cases of adverse events after vaccination should be notified to be properly investigated.Objective: To analyze the occurrence of post-vaccination adverse events in the municipality of Gurupi-TO. Methodology: This is a descriptive, documental study of a quantitative approach performed at the Municipal Health Department (SEMS) of Gurupi Tocantins. The target population of this study were the
${ }^{1}$ Discente do Curso de

Enfermagem da Universidade de Gurupi. Gurupi-TO, Brasil. E-mail:

alessandra.lauermann@hotmail .com

${ }^{2}$ Discente do Curso de Enfermagem da Universidade de Gurupi. Gurupi-TO, Brasil. E-mail:

karen_lohanny@hotmail.com

${ }^{3}$ Docente do Curso de

Enfermagem da Universidade de Gurupi.

E-mail:

soaresdenise468@gmail.com

Endereço para

correspondência:

Alessandra Luciane Lauermann

Rico. Rua Ministro Alfredo

Nasser $n^{\circ}$ 531, 77405130,

Gurupi-TO. 063984095755.

E-mail:

alessandra.lauermann@hotmail .com 
Adverse Post-Vaccination Events records of the Post-Vaccination Adverse Events Information System (SI-EAPV) of the cases occurred in the municipality of Gurupi in the period from 2011 to 2017 because they are available in the SEMUS archives. The sample consisted of 73 notification cards from post-vaccine adverse events (EAPV). Data were analyzed using descriptive statistics. Results: It was observed that the majority of notifications were of children under one year $36.99 \%$, and the female sex predominated among the notifications with $57.53 \%$. The combination of the VIP, Penta, VORH and Pnc10v vaccines was the one that caused the most adverse events $16.44 \%$, followed by the DT vaccine applied in isolation, representing $6.85 \%$ of the cases. The post-vaccine adverse events most reported during the study period were systemic fever $32.88 \%$ and nausea / vomiting $20.55 \%$, most were considered nonsevere and the vaccination schedule was maintained in $34.25 \%$ of the cases. Conclusion: It was concluded that although the immunobiologicals present benefits, they also present risks of PADV. However the effectiveness of the prevention that the vaccine provides outweighs the risks of having an adverse event provoked by it. This study will serve as a starting point for new studies in the state of Tocantins adding knowledge on the subject.

Descriptors: Vaccination. Immuno. Notification. Adverse reaction 


\section{INTRODUÇAO}

Com a descoberta da vacina, foi possível estabelecer como meta a imunização na população de forma que todos deveriam permanecer protegidos contra várias doençasimunopreveníveis. $A$ vacina pode ser apresentada isolada ou combinada e ainda apresentar diversos mecanismos de ação de acordo com os componentes antigênicos, sob forma de suspensão de bactérias vivas atenuadas, suspensão de bactérias mortas ou avirulentas, componentes das bactérias, toxinas obtidas em cultura de bactérias submetidas a modificações, vírus vivos atenuados, vírus inativados e fração de vírus. ${ }^{1} \mathrm{O}$ início da vacinação no país foi marcadopor revoltas e rejeição motivadas pela falta de conhecimento da população acerca dos benefícios das vacinas. Apesar da resistência da população, a vacina se tornou obrigatória para todos como forma deredução da incidência de algumas doenças. Nas últimas décadas é possível notar grandes avanços e significante redução na incidência de doenças infecciosas e parasitárias, resultado positivo da prática de controle por meio da vacinação. ${ }^{2-3}$

É certo que os imunobiológicos proporcionam inúmeros benefícios, no entanto não estão isentosde efeitos adversos e inesperados.Um evento adverso pós-vacinação pode ser compreendido como sendo algum sinal ou sintoma inesperado após a administração de algum tipo de imunobiológico. ${ }^{4}$ Os sinais ou sintomas causados podem se dividir em manifestações locais(eritema, calor, edema, enduração, nódulo, abscessos, dor localizada e etc.) ou sistêmicas ( anafilaxia, síncope, infarto do miocárdio,episódio hipotônico hiporresponsivo, choque séptico, broncoespasmo e etc. $)^{1} e$ ainda se classificar de acordo com a intensidade, sendo grave quando há necessidade de internação por no mínimo 24 horas podendo apresentar alguma disfunção ou sequela, moderado quando o indivíduo passa por uma consulta médica realizando exames e tratamento se necessário e leve quando não é necessária intervenção médica. Ainda podem ser classificados levando em consideração a causa, se induzido pela vacina por causa de seus componentes, erros programático ou coincidente, que significa que o evento já existia no momento da aplicação da vacinação e se manifestou apenas depois. ${ }^{3}$

A Organização Mundial de Saúde recomendou em 1991 que fosse empreendida uma maneira de estabelecer a vigilância sobre os eventos adversos pós-vacinação (EAPV). Em 1992 o Programa Nacional de Imunização começou a formar a estrutura de um Sistema Nacional de Vigilância de Eventos Adversos Pós-Vacinação que foi consolidado apenas em 2000 , com isso as informações lançadas neste sistema poderiam 
ser analisadas de forma consistente. Apenas em 1998 com a criação do Manual de Vigilância Epidemiológica dos Eventos Adversos pós-vacinação as notificações começaram a acontecer em todo o país, pois assim os profissionais obtiveram o conhecimento acerca da temática, e para reforçar a vigilância acerca dos EAPV foi lançada a portaria MS/GM n 1.271 que torna um agravo de notificação compulsória. ${ }^{5} \mathrm{~A}$ função da vigilância epidemiológica dos eventos adversos pós-vacinação é realizar o acompanhamento desses eventos para que os benefícios da vacinação sejam melhores que seus possíveis riscos.

De modo geral, as vacinas representam ser produtos biológicos altamente seguros, e levam benefícios inquestionáveis à saúde da população, porém como qualquer outro produto elas apresentam riscos de alguns EAPV que são esperados e outros não. ${ }^{6}$ Assim de acordo com as reações apresentadas os casos de eventos adversos pós-vacinação devem ser notificados para serem investigados. ${ }^{3}$ Diante do exposto este estudo teve como objetivo analisar a ocorrência de eventos adversos pós-vacinação no município de Gurupi-TO.

\section{MATERIAIS E METODOS}

Tratou-se de um estudo descritivo, documental de abordagem quantitativa realizado na Secretaria Municipal de Saúde(SEMUS) de Gurupi Tocantins. A população alvo deste estudo foram as fichas de Eventos Adversos Pós Vacinação do Sistema de Informação de Eventos Adversos Pós Vacinação (SI-EAPV) dos casos ocorridos no município de Gurupi no período de 2011 a 2017por serem apenas estes arquivados na SEMUS de Gurupi TOaté operíodo deste estudo. A coleta ocorreu na SEMUS pelas próprias pesquisadoras em abril de 2018.

A amostra constituiu-se de 73 fichas de notificação que foram disponibilizadas pela responsável pelo Programa de Imunização de Gurupi. Para a coleta dos dados foi utilizado um formulário contemplando as variáveis: idade, sexo, tipo de imunobiológico, evento adverso ocorrido.

Os critérios de inclusão adotados foram: casos notificados de eventos adversos pósvacinaçãoencontrados nos arquivos da SEMUS entre os anos de 2011 a 2017 e os critérios de exclusão foram as fichas de eventos adversos de anos diferentes ao período proposto pela pesquisapor apenas estas comporem o arquivo da SEMUS. 
Os dados coletados foram armazenados em um banco de dados construído a partir do programa Microsoft Office Excel 2010. Foram tratados por meio de estatística descritiva e demonstrados em tabelas. Esta pesquisa utilizou dados secundários o que dispensa a submissão ao Comitê de Ética em Pesquisa.

\section{DESENVOLVIMENTO}

Analisando as 73 fichas de notificações constatou-se que 27 casos (36,99\%)foram representados pela população de menores de 1 ano, 24 casos $(32,88 \%)$ eram de pessoas com idade entre 1 e 12 anos e 21 casos (28,77\%) representou a faixa etária acima de 25 anos. Nota-se grande representatividade das crianças menores de um ano, dado semelhante ao encontrado em outros estudos, sendo um em Teresina ${ }^{2}(\mathrm{PI})$ com 60 casos $(81,0 \%)$ e outro em Campo Grande ${ }^{7}$ com 13 casos $(31,7 \%)$, onde ambos demonstram a maior frequência em menores de um ano. Fato que pode estar relacionado com a quantidade de vacinas que o Programa Nacional de Imunização estabelece para essa faixa etária e a imaturidade do sistema imunológico. ${ }^{2}$

Tabela 1. Distribuição do número de casos de eventos adversos notificados de acordo com a faixa etária no município de Gurupi-TO de 2011 a 2017.

\begin{tabular}{cc}
\hline Faixa Etária & № de casos \\
\hline Menores de 1 ano & 27 \\
De 1 a 12 anos & 24 \\
Acima de 25 anos & 21 \\
Ignorado & 1 \\
Total & 73 \\
\hline
\end{tabular}

Fonte: Dados secundários.

Em relação ao sexo, 42 casos $(57,53 \%)$ corresponderam ao sexo feminino, informações corroboradaspelo estudo de Campo Grande ${ }^{7}$ que mostrou (63,4 \%).

Quanto aos imunobiológicos na maioria das fichas de notificação avaliadas não foi possível identificar qual o responsável pelo evento adverso, pois em muitos casos foram administrados até cinco imunobiológicos simultaneamente, ou ainda acredita-se que o mau preenchimento das fichas de notificações pode resultar em subnotificações. A combinação dos imunobiológicos VIP, PENTA, VORH, PNC10v foi a que gerou maior ocorrência de eventos adversos, (16,44\%) dos casos, o que difere de outros estudos 
como Teresina ${ }^{2}$, Campo Grande ${ }^{7}$ e Ceará ${ }^{8}$ ondea tetravalente prevaleceu $(63,0 \%)$, $(19,5 \%)$ e $(80,1 \%)$ respectivamente. Tal diferença possivelmente seja devido a inclusão da vacina Pentavalenteno calendário Nacional de Imunização apenas em 2012. Algumas das notificações com administração simultâneas de vacinas atribuíram as reações à vacina PENTA, devido ao componente Pertussis, principal componente da vacina responsável por causar reações. ${ }^{5} \mathrm{~A}$ vacina DT por sua vez aplicada de forma isolada também apresentou importante ocorrência (6,85\%) dos casos de eventos adversos encontrados.

Tabela 2. Distribuição percentual dos imunobiológicos que causaram eventos adversos no município de Gurupi-TO de 2011 a 2017.

\begin{tabular}{ccc}
\hline Imunobiológico & No total de casos & $\%$ \\
\hline VIP/Penta/VORH/PNC10v & 12 & 16,44 \\
DT & 5 & 6,85 \\
FA & 4 & 5,48 \\
Influenza & 4 & 5,48 \\
HB & 3 & 4,11 \\
HPV & 3 & 4,11 \\
BCG/HB & 3 & 4,11 \\
Penta & 2 & 2,74 \\
Outros & 37 & 50,68 \\
\hline
\end{tabular}

Fonte: Dados secundários.

Em relação às manifestações clínicas o estudo revelou que houve reações locais e sistêmicas. As reações sistêmicas se apresentaram com maior frequência, demonstradas pela febre $(32,88 \%)$ epela náuseas/vômitos $(20,55 \%)$ dos casos. Quanto às reações locais vislumbrou hiperemia $(17,88 \%)$, seguida pela dor $(15,07 \%)$ e pelo edema local $(13,70 \%)$.

No estudo de Teresina²também houve maior prevalência de febre $(76,0 \%)$. No entanto observou-se diferença nos estudos realizados em Minas Gerais ${ }^{9}$ e Ceará ${ }^{8}$ sendo o episódio hipotônico hiporresponsivo a manifestação mais frequente $(15,9 \%)$ e $(22,4 \%)$ respectivamente. 
Tabela 3. Distribuição percentual dos eventos adversos causados pelas aplicações dos imunobiológicos no município de Gurupi-TO de 2011 a 2017.

\begin{tabular}{ccc}
\hline Manifestações & № & $\%$ \\
\hline Febre & 24 & 32,88 \\
Náuseas e vômitos & 15 & 20,55 \\
Hiperemia & 13 & 17,81 \\
Dor & 11 & 15,07 \\
Edema & 10 & 13,70 \\
Choro & 7 & 9,59 \\
Calor & 6 & 8,22 \\
Convulsão & 6 & 8,22 \\
Outras & 79 & 108,22 \\
\hline
\end{tabular}

Fonte: Dados secundários.

\section{CONSIDERAÇÓES FINAIS}

Comeste estudo compreendeu-se que a prevenção de doenças imunopreveníveis proporcionadas pela imunização supera a ocorrência de eventos adversos produzidos por estas. Com o avanço da indústria farmacêutica as vacinas atuais causam cada vez mais menos eventos adversos. Supõe-se que a maioria dos eventos adversos ocorridos na amostra analisada possam estar relacionados a combinação de várias vacinas como preconiza o Calendário Nacional de Vacinação principalmente na faixa etária de maior ocorrência que foram crianças menores de um ano. Diante dos achados nesta pesquisa acredita-se que o enfermeiro deve se empenhar cada vez mais na vigilância e controle dos eventos adversos pós-vacinação.

Sugere-se que mais estudos como este seja realizado em todo o estado para se ter uma real compreensão da situação dos eventos adversos no estado do Tocantins.

\section{REFERENCIAS}

1. Brasil. Ministério da Saúde: Fundação Nacional de Saúde. Manual de Normas de Vacinação. 3.ed. Brasília: Ministério da Saúde, 2001.

2. Araújo TME, Carvalho PMG, Vieira EDF. Análise dos eventos adversos pós-vacinais ocorridos em Teresina. RevBrasEnferm. 2007 jul-ago;60(4):444-448.

3. Bissetto LHL, Cubas MR, Malucelli A. A prática de enfermagem frente aos eventos adversos pósvacinação. RevEscEnferm USP. 2011; 45(5):1128-1134. 
4. Alves H, Domingos LMG. Manejo de eventos adversospós-vacinação pela equipe de enfermagem: desafio parao cuidado.RevEnferm UERJ. 2013; 21: 502-507.

5. Brasil. Ministério da Saúde. Secretaria de Vigilância em Saúde. Departamento de Vigilância das Doenças Transmissíveis. Manual de vigilância epidemiológica de eventos adversos pós-vacinação. 3.ed. Brasília: Ministério da Saúde, 2014.

6. Brasil. Ministério da Saúde. Secretaria de Vigilância em Saúde. Departamento de Vigilância Epidemiológica. Manual de vigilância epidemiológica de eventos adversos pós-vacinação. 2.ed. Brasília: Ministério da Saúde, 2008.

7. Piacentini S, Contrera-Moreno L. Eventos adversos pós-vacinais no município de Campo Grande (MS, Brasil). CienSaude Colet. 2011 jan-fev; 16(2): 531-536.

8. Costa AS, Braga AVL, Bastos ECSA, Lima GG, Chaves ES. Vigilância de eventos adversos pósvacinação no estado do Ceará, em 2011. EpidemiolServSaude. Brasília. 2015 jan-mar; 24(1):155-160.

9. Silva SS, Oliveira VC, Ribeiro HCTC, Alves TGS, Cavalcante RB, Guimarães EAA. Análise dos eventos adversos após aplicação de vacinas em Minas Gerais, 2011:um estudo transversal.EpidemiolServSaude, Brasília.2016 jan-mar; 25(1):45-54. 